\title{
Optimization of Agrobacterium tumefaciens-mediated transformation and shoot regeneration after co-cultivation of cabbage (Brassica oleracea subsp. capitata) cv. KY Cross with AtHSP101 gene
}

\begin{abstract}
A number of parameters which have been reported to influence genetic transformation via Agrobacterium-mediated transformation method were evaluated to increase the frequency of transformation of cabbage (Brassica oleracea subsp. capitata) cv. KY Cross with AtHSP101 gene. The binary vector pCAMHSP was designed and mobilized into two Agrobacterium tumefaciens strains C58 and GV2260. The study was carried out on hypocotyl and shoot tip explants of cabbage cv. KY Cross. Transformation parameters optimized were pre-culture medium, acetosyringone application, bacterial density and inoculation time. The polymerase chain reaction (PCR) assay and production of mRNA of AtHSP101 gene were confirmed by reverse transcription-polymerase chain reaction (RT-PCR). The expression of LacZ gene in the transgenic plants also showed that it could be applied as a plant transformation reporter gene in genetic transformation studies. Multiple shoot regeneration of hypocotyl and shoot tip explants of cabbage after co-cultivation with Agrobacterium was optimized and medium containing $2 \mathrm{mg} / \mathrm{L}$ BAP was observed to be the best for shoot regeneration after cocultivation. In this study, $45 \%$ and $32.5 \%$ transformation efficiencies were achieved for hypocotyl and shoot tip explants, respectively using the optimized procedure.
\end{abstract}

Keyword: Agrobacterium tumefaciens, Cabbage, LacZ gene, Regeneration, Transformation 